\title{
Super-resolution Ultrasound Imaging Scheme Based on a Symmetric Series Convolutional Neural Network
}

This paper was downloaded from TechRxiv (https://www.techrxiv.org).

\section{LICENSE}

CC BY 4.0

SUBMISSION DATE / POSTED DATE

28-07-2021 / 28-07-2021

\section{CITATION}

Tamang, Lakpa Dorje (2021): Super-resolution Ultrasound Imaging Scheme Based on a Symmetric Series Convolutional Neural Network. TechRxiv. Preprint. https://doi.org/10.36227/techrxiv.15066315.v1

DOI 


\title{
Super-resolution Ultrasound Imaging Scheme Based on a Symmetric Series Convolutional Neural Network
}

\author{
Lakpa Dorje Tamang and Byung Wook Kim, Member, IEEE
}

\begin{abstract}
In this paper, we propose a symmetric series convolutional neural network (SS-CNN), which is a novel deep convolutional neural network (DCNN)-based superresolution (SR) technique for ultrasound medical imaging. The proposed model comprises two parts: a feature extraction network (FEN) and an up-sampling layer. In the FEN, the low-resolution (LR) counterpart of the ultrasound image passes through a symmetric series of two different DCNNs. The low-level feature maps obtained from the subsequent layers of both DCNNs are concatenated in a feed forward manner, aiding in robust feature extraction to ensure high reconstruction quality. Subsequently, the final concatenated features serve as an input map to the latter 2D convolutional layers, where the textural information of the input image is connected via skip connections. The second part of the proposed model is a sub-pixel convolutional (SPC) layer, which up-samples the output of the FEN by multiplying it with a multi-dimensional kernel followed by a periodic shuffling operation to reconstruct a high-quality SR ultrasound image. We validate the performance of the SS-CNN with publicly available ultrasound image datasets. Experimental results show that the proposed model achieves an exquisite reconstruction performance of ultrasound image over the conventional methods in terms of peak signal-to-noise ratio (PSNR), and structural similarity index (SSIM), while providing compelling SR reconstruction time.
\end{abstract}

Index Terms-ultrasound imaging, image enhancement, super resolution, neural network, deep learning

\section{INTRODUCTION}

$\mathrm{I}$ MAGE super resolution (SR) [1, 2] refers to the reconstruction of a high-resolution (HR) image from its counterpart in the low-resolution (LR) space. Recently, SR image reconstruction has been a prolific area of research in the fields of digital image processing and computer vision because of its ability to solve the inherent resolution limitation problems of low-cost image sensors. Real-world applications for SR range from enhancement of blurred and noisy images/videos into high-definition (HD) images/videos [3], robust pattern recognition [4], and microscopic object detection [5]. In addition, a higher-quality image obtained from SR leads to a higher degree of accuracy in medical imaging analysis, where proper and accurate localization of tumors is required.
Generally, the hardware limitations of high-end medical imaging devices make it difficult to identify the abnormalities of internal organs and the underlying anatomy and tissues [5]. Because poor visual quality in medical images imposes a limitation on posterior analysis of medical conditions, and can eventually lead to false suppositions, there is an extensive demand for the SR technique in medical imaging. Within the medical imaging domain, ultrasound is a versatile and widely practiced diagnostic tool. Compared to other medical imaging modalities, ultrasound usually produces poor spatial resolution of deep tissues due to the wavelength-dependent inverse relation between penetration depth and resolution [6]. Therefore, exploitation of the SR task to effectively increase the resolution of poor-quality ultrasound images has recently prompted extensive interest of research $[7,8]$.

In previous research, multiple SR studies based on interpolation techniques $[9,10]$, and sparse representation $[11$, 12] have been explored for enhancing the quality of images to the desired resolution. Recently, due to the paradigm shift in image processing technology, the deep learning (DL) framework has been considered for SR tasks [13-16] in various applications. These DL techniques $[17,18]$ offer the significant prospect of providing better SR quality than conventional schemes because of their ability to learn non-linear feature representatives from image data. Popular frameworks such as ResNet [19], DenseNet [20], and recurrent neural networks (RNNs) [21] have been utilized in SR tasks with natural images. Particularly in the medical imaging domain, multiple studies [5, 22-24] have investigated DL-based SR approaches. However, a limited number of studies [25-27] have been conducted by incorporating DL technology to enhance the quality of ultrasound images. Liu et al. [25] proposed a perception consistency ultrasound image SR technique based on a selfsupervised cycle generative adversarial network (CycleGAN) framework. This study integrated multi-level feature loss and the adversarial characteristics of the generator during the SR task to balance the visual similarity between real data and reconstructed data. In [26], a multi-frame SR approach was proposed for ultrasound images from a set of LR images. To cope with motion estimation while performing multi-frame SR, this research proposed a DL network that obtains HR images by

This research was supported by National Research Foundation of Korea (NRF) grant funded by the Korean government (NRF2019R1A2C4069822).

L. D. Tamang and B. W. Kim are with the Department of Information and Communication Engineering, Changwon National University, Uichanggu, Changwon-si, 51140, South Korea (e-mail: l.tamang9@changwon.ac.kr, bwkim@changwon.ac.kr (corresponding author)). 
reducing the effect of existing noise in LR ultrasound images. Similarly, in [27], a fast medical image super-resolution (FMISR) method was proposed where a set of three hidden layers with a mini-network in between are used for complete feature extraction. This is followed by a sub-pixel convolution (SPC) layer for successful image up-sampling. Although these techniques yielded convincing results in terms of image quality, they do not exploit the low-level features of the input image throughout the network. The need for low-level features while implementing any SR task is crucial, because it can provide additional information for reconstructing the high-frequency details of the HR image [28]. In addition, when low-level features are passed into the latter layers through skip connections, the vanishing gradient problem can be alleviated. Furthermore, conventional techniques do not take feature redundancy into account, which instigates the risk of learning the same feature multiple times, resulting in limited reconstruction quality of the SR imaging.

To overcome these shortcomings, we propose a novel SR model for ultrasound imaging, referred to as a symmetric series convolutional neural network (SS-CNN). The proposed model comprises two parts: a feature extraction network (FEN) and up-sampling. To extract vital features that contain fine structural details of ultrasound images, a FEN consisting of a symmetric series of two feed forward convolutional neural networks (CNNs) was designed. Subsequent layers in the SSCNN are concatenated with skip connections to utilize lowlevel features of the LR input image and to minimize the vanishing gradient problem. This leads to reconstruction of rich high-frequency details from the input image while conducting the SR task. In addition, it provides the benefits of the compact network structure with minimal feature redundancy by allowing feature reuse between symmetric convolutional series. In doing so, a considerable number of features, including details of highfrequency components, will be propagated to the final layer of the FEN. Then, the up-sampling procedure is conducted by employing the SPC layer as an up-sampling operator. In this layer, the feature map generated by the FEN is multiplied by a multi-dimensional kernel and then subjected to a periodic shuffling operation to finally generate the output HR image. In the test results from using a publicly available ultrasound image database, we observed that the proposed scheme offered superior performance in terms of peak signal-to-noise ratio (PSNR), structural similarity index (SSIM), and a fair SR reconstruction speed, compared to state-of-the-art SR approaches.

The rest of the paper is organized as follows. In Section II, the proposed system is discussed. Experiments and results are discussed in Section III. Finally, in Section IV, conclusions are reported.

\section{THE PROPOSEd SYSTEM}

The system architecture of the proposed scheme is depicted in Fig. 1. The network is comprised of the FEN and an upsampling layer. The FEN is responsible for extracting imminent features from the input image and forwarding them to the upsampling layer. The up-sampling layer then enhances image resolution by upscaling the output feature map from the preceding layer to produce the HR output.

\section{A. The Feature Extraction Network}

Feature extraction in SR tasks can be formulated as a sampling problem where perfect reconstruction of the desired features is required. The main purpose of this network is to extract image blocks from LR images; the dimensions of the extracted blocks remain in the LR space. This network basically extracts local hierarchical features of an input LR image in different stages, from shallow to deep. To acquire more hierarchical representations of the input image, increasing the depth of the FEN is the general approach in the DL paradigm. The deep layers can prominently extract various ranges of useful feature representations (low to high); however, the huge gradient loss from the backpropagation process must be dealt with. Therefore, an alternative structure for building a FEN would be passing the same input to a series of two symmetric convolutional layers, where the feature maps of the corresponding layers are concatenated. With the symmetric series, abundant semantic information of the LR input can be explored, from which better perceptual quality of the SR output can be achieved. Note that the resolution of ultrasound images is impeded by the fundamental limits of diffraction, creating a long-standing trade-off between resolution and penetration. Accurate image reconstruction is therefore crucial for the SR task, and can be achieved by using a robust feature extractor prior to up-sampling.

In the SS-CNN model, the FEN consists of a symmetric series of two convolutional layers: Conv A and Conv B. Each series holds $M$ convolutional layers ( $M$ being the depth of each series) followed by a rectified linear unit (ReLU) as a non-linear activation function. The input for the network is the LR samples created by down-sampling the original HR samples. During forward propagation, the feature maps generated by subsequent Conv A and Conv B layers are concatenated. This allows the FEN to learn global features of the ultrasound images by concealing together the local features of both inputs from each subsequent layer. In this way, the network avoids learning redundant features and instead learns a unique representation of the input produced by each layer. The network also facilitates sufficient regularization as the weights are shared between each symmetric convolutional layer, so any external regularization techniques are not required. Besides, skip connections are introduced between the input image and the latter convolutional layers of the FEN. By doing so, the preliminary features of the input image are directly bypassed to the final layers, and the original textural information of the image can be preserved. The skip connections also help to alleviate the problem of gradients vanishing from the DCNNs [29].

The detailed structure of the proposed FEN with $M=6$ is presented in Table I. The convolutional layers of both series use 64 filters and $3 \times 3$ kernel sizes to convolve over an image during single convolution. Small-sized filters are preferred because they facilitate easier weight sharing and reduce the network complexity. The cumulative features extracted by concatenating simultaneous feature maps from both series are forwarded to two convolutional layers at the latter stages of feature extraction. The later layers use thirty two, and three 


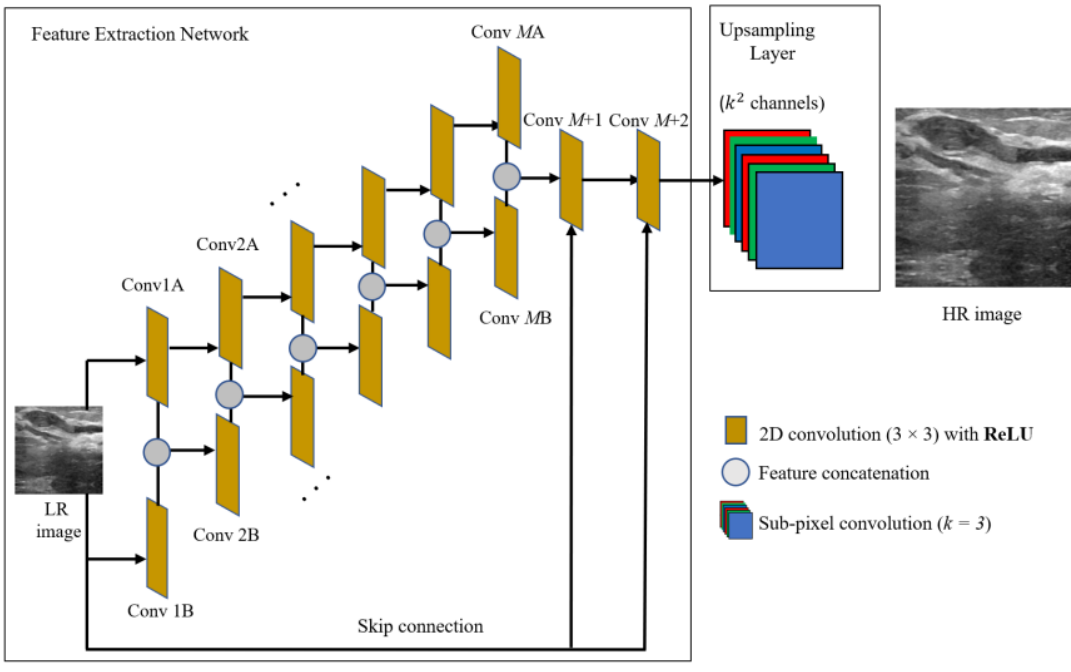

(a)

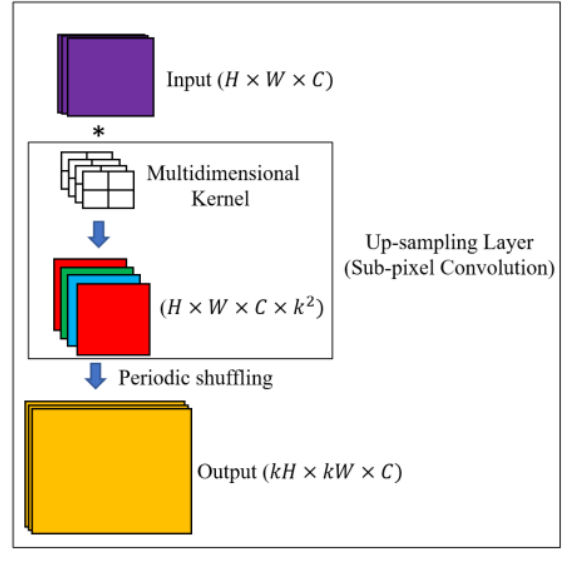

(b)

Fig. 1. Schematic representation of the SS-CNN model. (a) Feature extraction network, and (b) Up-sampling layer

TABLE I

NETWORK DETAILS

\begin{tabular}{cccccc}
\hline Layers & Input & Filters & $\boldsymbol{k}$ & Kernel size & Output \\
\hline Conv1A & $(100,100,3)$ & 64 & - & $3 \times 3$ & $(100,100,32)$ \\
Conv1B & $(100,100,3)$ & 64 & - & $3 \times 3$ & $(100,100,32)$ \\
Conv2A & Conv1A & 64 & - & $3 \times 3$ & $(100,100,32)$ \\
Conv2B & Concat (Conv 1A, Conv1B) & 64 & - & $3 \times 3$ & $(100,100,32)$ \\
Conv3A & Conv2A & 64 & - & $3 \times 3$ & $(100,100,32)$ \\
Conv3B & Concat (Conv 2A, Conv2B) & 64 & - & $3 \times 3$ & $(100,100,32)$ \\
Conv4A & Conv3A & 64 & - & $3 \times 3$ & $(100,100,32)$ \\
Conv4B & Concat (Conv 3A, Conv3B) & 64 & - & $3 \times 3$ & $(100,100,32)$ \\
Conv5A & Conv4A & 64 & - & $3 \times 3$ & $(100,100,32)$ \\
Conv5B & Concat (Conv 4A, Conv4B) & 64 & - & $3 \times 3$ & $(100,100,32)$ \\
Conv6A & Conv5A & 64 & - & $3 \times 3$ & $(100,100,32)$ \\
Conv6B & Concat (Conv 5A, Conv5B) & 64 & - & $3 \times 3$ & $(100,100,32)$ \\
Conv7 & Concat (Conv 6A, Conv6B) & 32 & - & $1 \times 1$ & $(100,100,16)$ \\
Conv8 & Conv 7 & 3 & - & $1 \times 1$ & $(100,100,3)$ \\
Up-sampling layer & Conv 8 & - & 3 & - & $\left(100,100,3 \times 3^{2}\right)$ \\
Periodic shuffling & $\left(100,100,3 \times 3^{2}\right)$ & - & - & - & $(300,300,3)$ \\
\hline
\end{tabular}

filters respectively with a receptive size of $1 \times 1$. To achieve potential improvement in SR reconstruction performance, both later layers are concatenated with the input image via skip connections. This ensures that the final layer of the feature extraction network contains enough textural information from the input image.

Note that the design of this network focuses on extracting global features from the input image. Therefore, to achieve this, the convolutional layers from both series have an identical size for the receptive field so the concatenated feature maps from the subsequent layers of Conv A and Conv B accommodate sufficient image information. In addition, to preserve important details of the feature maps produced, pooling and downsampling layers are not used throughout the network.

\section{B. Up-sampling Layer}

The up-sampling layer is responsible for reconstructing a high-quality HR image from the image features extracted from the FEN. Generally, in conventional DL-based SR tasks, a transposed convolution (deconvolution) layer is used for upsampling to reconstruct high-quality images [13]. However, since the FEN of the proposed model does not utilize any downsampling layers (e.g., maxpooling), the transposed convolution layers are not suitable candidates for an up-sampling operation. Moreover, using the deconvolutional layer for up-sampling has the disadvantage of significantly increasing the computational cost and model complexity due to the large numbers of feature maps being fed into it. Therefore, to up-sample the features of an LR image into the high-quality HR space, we used the SPC layer, which is a learnable layer that first multiplies its input 
with a multi-dimensional kernel to generate a pool of channels for up-sampling. This pool of channels is then rearranged to generate output with a significantly higher resolution. With this, the network can learn to use multiple channels of the LR image features obtained from the FEN to represent a single HR image. Unlike the deconvolutional layer, which explicitly enlarges the feature map to increase resolution, the SPC layer expands the number of feature maps and applies a specific region-mapping criterion to obtain the HR output. From this, there is uniformity in the resolution of the feature maps obtained from the FEN, and the model becomes computationally less expensive.

In the SPC layer, the sets of weights for the convolutional kernel are independent from each other during convolution. For each feature map, this layer can generate $k^{2}$ channels in a single upscaling, where $k$ is the upscale factor. Therefore, by choosing the desired value of $k$, the SPC layer can map the image to the HR space from its LR counterpart in a single upscaling. From Fig. 1(b), we can clearly see that by convolving the input of dimensions $H \times W \times C$ (height, width, and channels, respectively) with a multi-dimensional kernel, we can obtain output with the same dimensions as the input but with additional $k^{2}$ channels. Subsequently, a periodic shuffling (PS) operation is used to reshape the output channels to the desired HR output. This operation rearranges a tensor with dimensions $\left(H \times W \times C . k^{2}\right)$ into a $(k H \times k W \times C)$ matrix. From this, $k^{2}$ channels are distributed to the spatial dimension of the image, where both height and width are multiplied by $k$ to generate the HR output. Throughout this process, the up-sampling layer is able to generate HR output with a single upscaling of the feature maps obtained from the FEN without using a complex and computationally expensive deconvolution operation.

\section{Datasets}

The proposed model was evaluated using two publicly available ultrasound image datasets, with examples as shown in Fig. 2. The breast ultrasound images (BUSI) dataset [31] (Dataset A) was collected in 2018 at Baheya Hospital, Cairo, Egypt, for early detection and treatment of women's cancer. A total of 780 images with an average resolution of $500 \times 500$ pixels were acquired from female candidates between ages 25 and 75 years. The dataset consists of 133 normal images
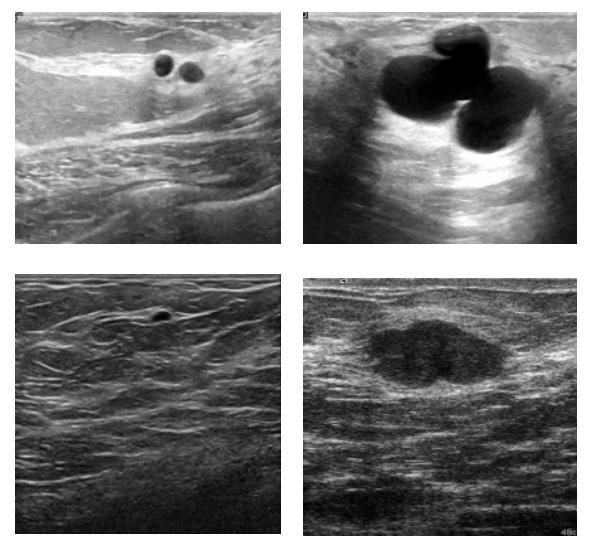

Fig. 2. Image samples for training: top row, from Dataset $A$, and bottom row, from Dataset $B$. (without cancer), 437 images with cancer, and 210 images with benign tumors. The whole dataset was divided into three parts: training with 521 images, validation using 109 images, and testing 150 images.

The other dataset [32] (Dataset B) was collected from the UDIAT Diagnostic Center of the Parc Tauli Corporation in Sabadell, Spain. This dataset was collected in 2012 using an 8.5 $\mathrm{MHz}$ Siemens ACUSON Sequoia C512 HD linear array transducer. The dataset contains a total of 163 images with a mean resolution of $760 \times 570$ pixels, where 53 are cancer images, and the remaining 110 are images of benign lesions. The main purpose for creating this dataset was detecting the lesions; however, in this research we employed it for the SR task.

\section{Data Augmentation}

The images available from the datasets were not numerous enough to prevent overfitting and for properly training various weights of the SS-CNN structure. Therefore, we expanded the dataset's limited availability by adapting a data augmentation technique. In the experiment, various augmentation techniques, such as rescaling, rotating, horizontal and vertical shifting, horizontal flipping, and zooming, were utilized. During data augmentation, we increased the dataset sizes by adding synthetic instances to the existing training set. These instances were created by applying domain-specific techniques, such as geometric transformations, to the original samples. Dataset A and Dataset B were augmented to obtain 880 and 862 images for training, respectively.

TABLE II NETWORK PARAMETERS

\begin{tabular}{cc}
\multicolumn{2}{c}{ NETWORK PARAMETERS } \\
\hline Parameter & Value \\
\hline Loss function & Mean squared \\
& error (MSE) \\
Optimizer & Adam \\
Learning rate & 0.001 \\
Training epochs & 100 \\
\hline
\end{tabular}

\section{E. Training}

For the SR task, the training process follows a selfsupervised learning strategy in which manual labelling of the training data is not required. The LR images are first generated by down-sampling their HR counterparts from the training sets. The output generated during forward propagation was compared with the original HR images to compute the loss function. The parameters used while training the network are presented in Table II. The whole network was optimized by minimizing the mean square error (MSE) loss between the model's prediction and its discrete HR ground truth images. For SR imaging, the previous study in [30] showed robust image quality with the adaptive momentum (Adam) optimizer. Therefore, to handle sparse gradients due to the noisy spatial resolution of LR images, we used the Adam optimizer with a learning rate of 0.001 . During training, the model learns at the level of individual image pixels. To cope with the input image dimensions for the designed network, the images were resized to $300 \times 300$ pixels. For training and validation purposes, $80 \%$ and $20 \%$ of the dataset images were respectively allocated. The whole model was trained within 100 epochs with a smaller 


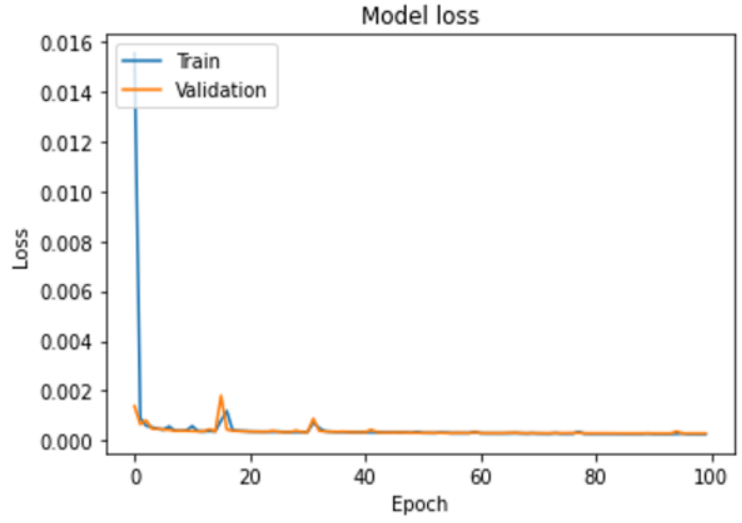

(a)

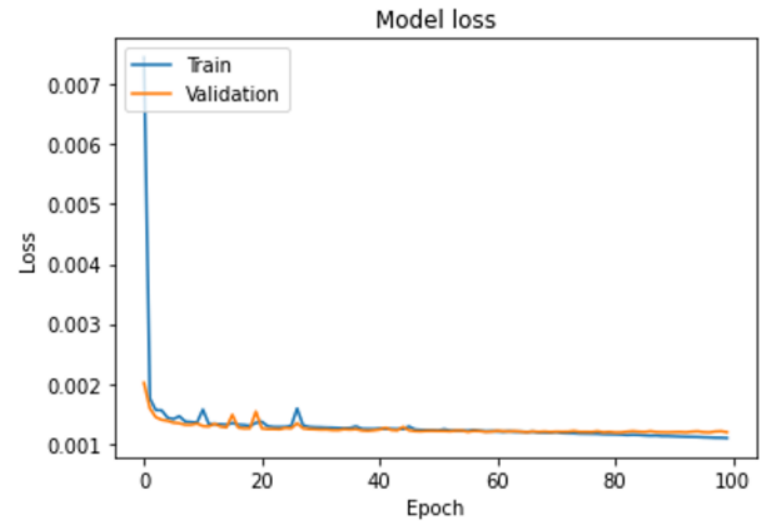

(b)

Fig. 3. Learning curves from training on the validation datasets: (a) dataset $A$, and (b) dataset $B$.

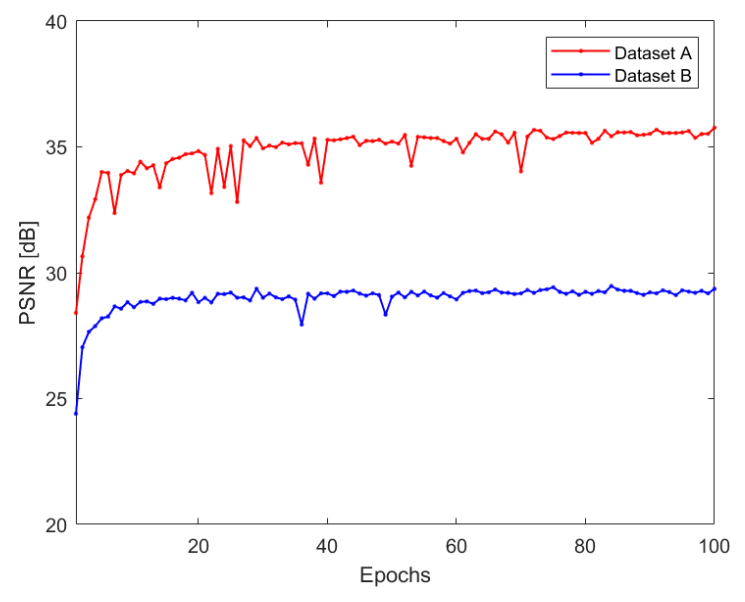

Fig. 4. PSNR vs. epochs of the SS-CNN model from the different datasets.

batch size of 8 to improve generalization of the proposed model. Figs. 3(a) and (b) show the learning curves of the SR model with datasets $\mathrm{A}$ and $\mathrm{B}$, respectively, for both training and validation datasets. In both scenarios, the training and validation loss converge with respect to epochs. No sign of overfitting can be observed, which demonstrates effective training of the model up to the point where saturation in training loss and accuracy was achieved.

Furthermore, the SR model learns to enhance the visual quality of the LR image as the training continues, and therefore, PSNR increments relative to the training epochs are shown in Fig. 4. For Dataset A, the PSNR increases from $28 \mathrm{~dB}$ and finally reaches $35 \mathrm{~dB}$. Here, we can observe saturation of PSNR after 70 epochs. In contrast, for Dataset B, the PSNR during the initial stage of training was $24 \mathrm{~dB}$, which finally reached $29 \mathrm{~dB}$ during the final epochs. This indicates that loss in the model converges with the training epochs, and therefore, the model was trained properly on both datasets. Fig. 5 shows the reconstructed images at the beginning and the end of the training phase. As shown in Figs. 5(b) and (d), the reconstructed image presents an enhanced image quality and valuable details.

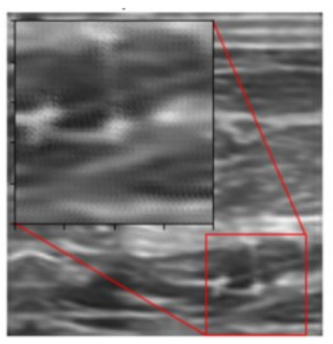

(a)

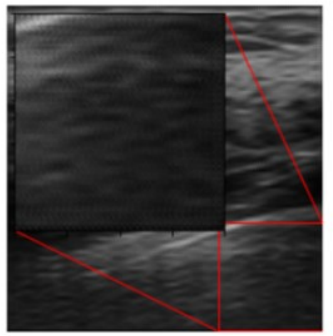

(c)

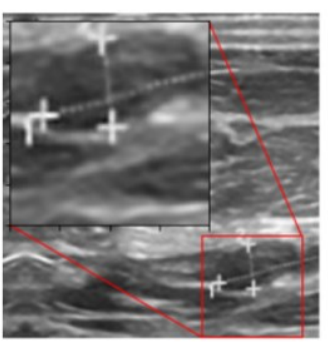

(b)

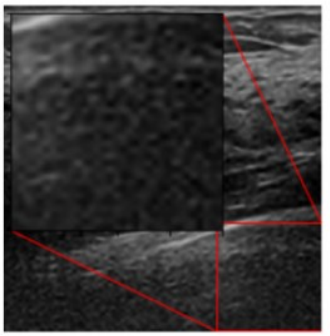

(d)
Fig. 5. Reconstructed images at the beginning and the end of the training phase: (a) Dataset A: HR at epoch 1 (28.40 dB), (b) Dataset A: HR at epoch 100 (35.76 dB), (c) Dataset B: HR at epoch $1(24.40 \mathrm{~dB})$, and (d) Dataset B: HR at epoch $100(29.36 \mathrm{~dB})$.

A significant difference in the visual quality of the image can be observed between the first and final epochs, thus, we can consider that the model we proposed to have learned the given datasets well.

\section{EXPERIMENTS AND RESULTS}

This section addresses the performance evaluation of the proposed model by analyzing reconstructed SR images from publicly available ultrasound imaging datasets. The whole experiment was performed on an AMD Ryzen processor at 3.7 GHz with $32 \mathrm{~GB}$ of RAM and an RTX $2080 \mathrm{Ti}$ GPU running Windows 10 and using Keras 2.3.1 with a TensorFlow 1.15 library in a Python (version 3.6) environment. This section 


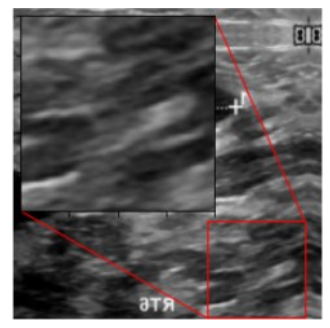

Tr
Ground truth

Bicubic

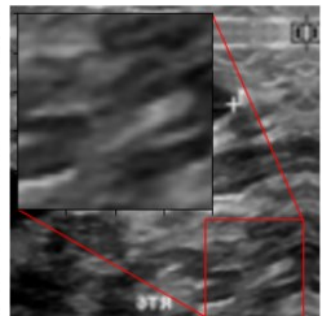

$30.64 \mathrm{~dB} / 0.8965$
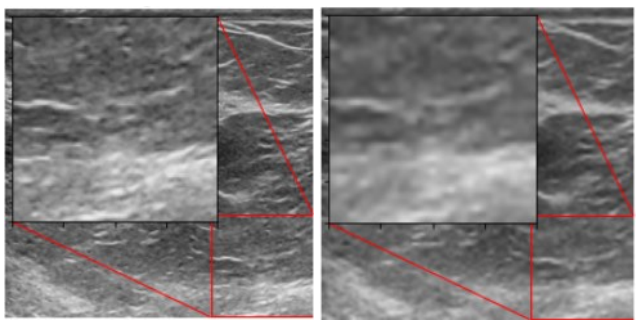

$26.79 \mathrm{~dB} / 0.8326$
ESPCN

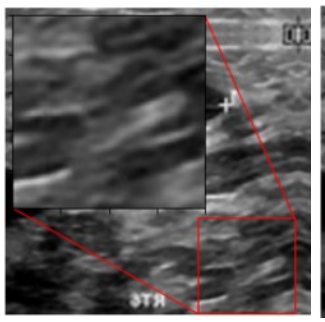

$32.56 \mathrm{~dB} / 0.9189$

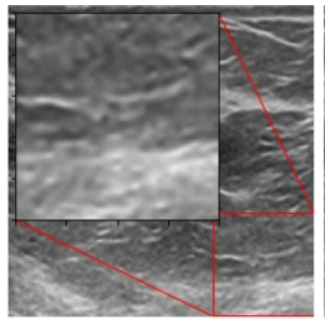

$27.96 \mathrm{~dB} / 0.8802$
FMISR

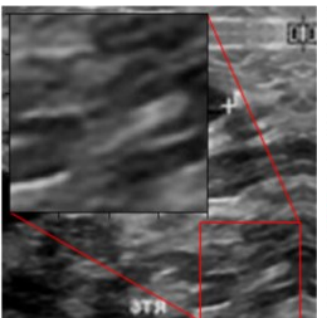

$32.24 \mathrm{~dB} / 0.9156$

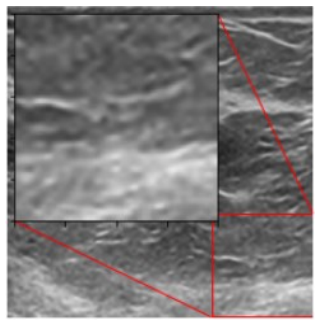

$27.83 \mathrm{~dB} / 0.8766$
SS-CNN

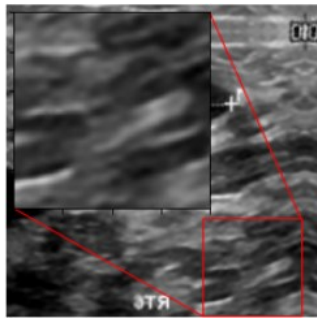

$33.05 \mathrm{~dB} / 0.9292$

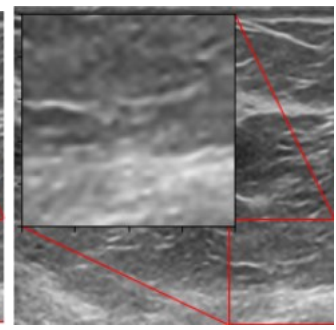

$28.27 \mathrm{~dB} / 0.8898$

Fig. 6. Ground truth HR image and reconstructed HR images generated from various reference methods including the proposed model. PSNR and SSIM values are shown below each sub-figure. Top row (Dataset A), and bottom row (Dataset B). Bold characters represent the highest value of PSNR and SSIM.

has a detailed discussion about the comparison between the proposed SR model and existing state-of-the-art approaches with respect to image quality and reconstruction speed.

\section{A. Visual Assessment}

The visual quality of the SR images was assessed based on image distortion and noise level during reconstruction. In this regard, PSNR and SSIM were used for comparing HR image restoration results. The former measures the ratio between the maximum possible value of the signal and the power of the noise that affects the quality of its representation. The latter is based on factors such as luminance, contrast, and structure that are appropriate for the human visual system. For the performance comparison, we evaluated the proposed SS-CNN model, bicubic interpolation, and two other state-of-the-art methods: ESPCN [13], and FMISR [23]. For the up-sampling procedure after feature extraction, the ESPCN SR model also utilizes an SPC technique. Note that we evaluated these models on top of publicly available ultrasound image datasets [25], [26], and thus, the reported values might slightly deviate from those presented in the original paper, which were obtained from different datasets.

Fig. 6 presents a visual quality assessment of the HR images reconstructed using the bicubic method, two state-of-the-art DL-based SR methods (ESPCN [13], and FMISR [25]), and the proposed SS-CNN model. We can see that the bicubic method exhibits the worst performance, where an excessive amount of visual artifacts, such as blurriness and image noise, can be observed. The HR images reconstructed with ESPCN [13] and FMISR [25] endured comparatively fewer visual artifacts than the bicubic method. However, their feature extraction capability was limited, and thus, these techniques failed to preserve the high-frequency details of the images. We can see that, of all the other methods, the SS-CNN model provided the best visual quality in the reconstructed HR images. This was validated by the PSNR and SSIM values, which are significantly higher than those provided by the reference schemes. The visual perception and textural patterns of the obtained HR ultrasound images avoided large distortions when compared to the input LR images. These convincing results can be attributable to the symmetric network structure of the FEN, with subsequent feature concatenations to extract significant spatial features from the pixel space. The concatenation of subsequent features from each layer of the symmetric convolutional network series allowed preserving the vital spatial and textural information

TABLE III

QuANTITATIVE ANALYSIS OF THE PROPOSED SR MODEL WITH CURRENT STATE-OF-ART AlgORITHMS

\begin{tabular}{cccccc}
\hline Dataset & $\boldsymbol{k}$ & Methods & $\begin{array}{c}\text { Mean } \\
\text { PSNR } \\
{[\mathrm{dB}]}\end{array}$ & $\begin{array}{c}\text { Mean } \\
\text { SSIM }\end{array}$ & $\begin{array}{c}\text { SR } \\
\text { testing } \\
\text { time }\end{array}$ \\
\hline & 3 & Bicubic & 31.14 & 0.8861 & - \\
$\mathrm{A}$ & 3 & $\begin{array}{c}\text { ESPCN } \\
{[13]}\end{array}$ & 32.83 & 0.9024 & $0.326 \mathrm{~s}$ \\
& 3 & $\begin{array}{c}\text { FMISR } \\
{[25]}\end{array}$ & 32.56 & 0.9011 & $0.255 \mathrm{~s}$ \\
& & SS-CNN & $\mathbf{3 3 . 4 4}$ & 0.9178 & $0.310 \mathrm{~s}$ \\
\hline
\end{tabular}

\begin{tabular}{cccccc}
\hline Dataset & $\boldsymbol{k}$ & Methods & $\begin{array}{c}\text { Mean } \\
\text { PSNR } \\
{[\mathbf{d B}]}\end{array}$ & $\begin{array}{c}\text { Mean } \\
\text { SSIM }\end{array}$ & $\begin{array}{c}\text { SR } \\
\text { testing } \\
\text { time }\end{array}$ \\
\hline B & 3 & $\begin{array}{c}\text { Bicubic } \\
\text { ESPCN } \\
{[13]}\end{array}$ & 30.31 & 0.8328 & - \\
& 3 & $\begin{array}{c}{[13]} \\
\text { FMISR } \\
{[25]}\end{array}$ & 31.17 & 0.8643 & $0.332 \mathrm{~s}$ \\
& 3 & SS-CNN & $\mathbf{3 1 . 7 9}$ & 0.8653 & $0.296 \mathrm{~s}$ \\
\hline
\end{tabular}




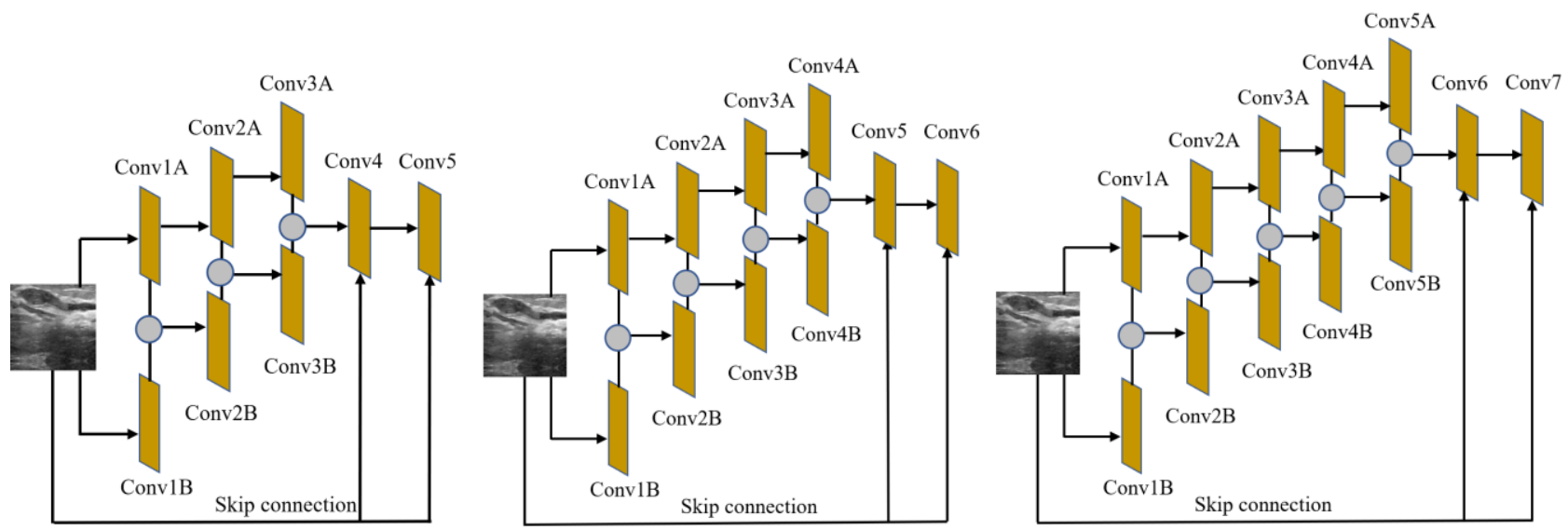

(a)

(b)

(c)

Fig. 7. FEN with varying depth. (a) $M=3$, (b) $M=4$, and (c) $M=5$

LR
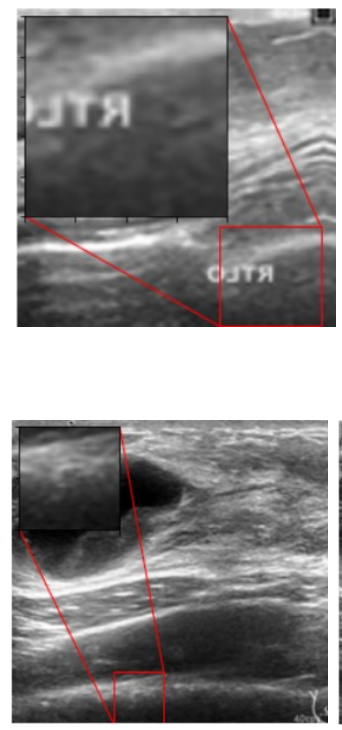

$M=3$

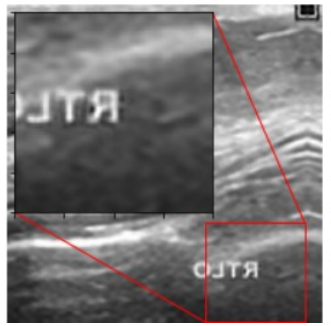

$31.16 \mathrm{~dB} / 0.9148$

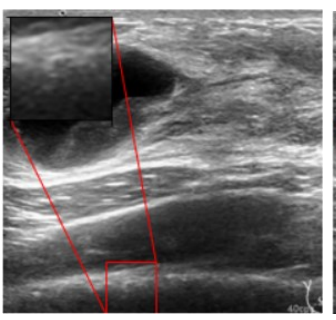

$28.84 \mathrm{~dB} / 0.8741$

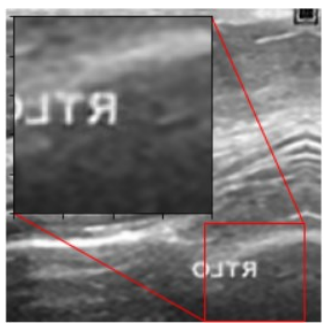

$31.53 \mathrm{~dB} / 0.9154$

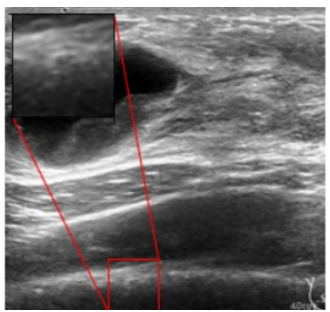

$30.17 \mathrm{~dB} / 0.8770$
$M=5$

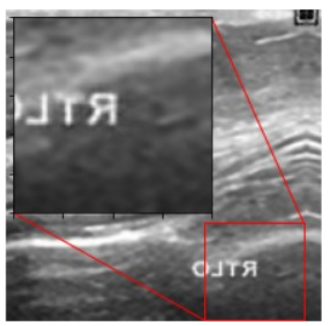

$31.47 \mathrm{~dB} / 0.9153$

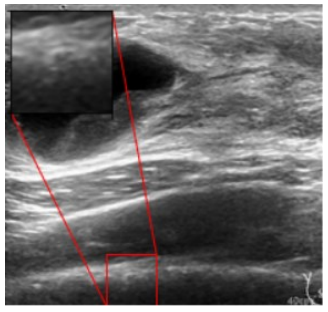

$29.97 \mathrm{~dB} / 0.8758$
$M=6$

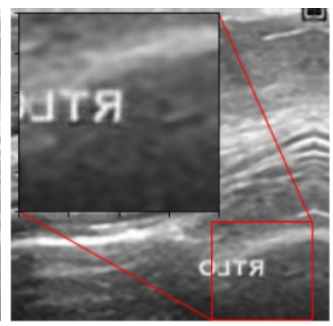

$31.17 \mathrm{~dB} / 0.9134$

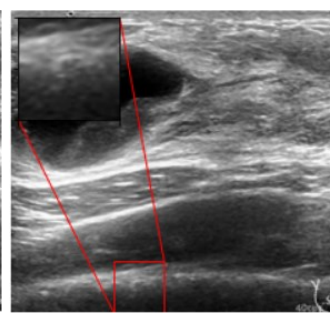

$29.60 \mathrm{~dB} / 0.8756$

Fig. 8. SR results produced by the FEN with variable network depths for Dataset A (top row), and Dataset B (bottom row). PSNR and SSIM values are shown below each sub-figure. Top row (Dataset $A$ ), and bottom row (Dataset $B$ ). Bold characters represent the highest values of PSNR and SSIM.

from the input image, thereby yielding less blurry HR images with relatively minimal visual artifacts. Moreover, the combination of features from the input image with high-level features during the latter stage of the feature extraction network via skip connections provided complementary information from the original image to enhance the HR output.

Table III summarizes the quantitative results where the proposed SS-CNN model is compared with state-of-the-art DLbased SR models. For performance comparisons, the image quality of the reconstructed HR image and the time consumed for HR image reconstruction were assessed. Mean values for PSNR and SSIM were recorded for all the test images, which were unseen during training. For SR testing time, we recorded the total time taken for the LR image to be converted into its corresponding HR counterpart. It can be observed that for both datasets, PSNR and SSIM provided by the SS-CNN outperformed all the reference algorithms. In particular, the SSCNN model achieved a PSNR improvement of 2.3, 0.61, and $0.88 \mathrm{~dB}$, respectively, from bicubic interpolation, and ESPCN and FMISR state-of-the-art SR techniques with Dataset A. With Dataset B, the SS-CNN model improved the PSNR by 1.48 , 0.17 , and $0.62 \mathrm{~dB}$, respectively. Similarly, the SSIM value was incremented by $0.0317,0.0154$, and 0.0167 with Dataset A and by $0.0325,0.001$, and 0.0031 with Dataset $\mathrm{B}$, when compared to bicubic interpolation, ESPCN, and FMISR, respectively. As mentioned, the significant PSNR gain was achieved due to the exquisite feature extraction network that retained the robust features necessary for up-sampling from the spatial regions of 
the input LR image.

Note that improving the reconstruction time in the SR task is as important as increasing the quality of the reconstruction in order for pathologists to make prompt clinical decisions. We can see from Table IV that the SS-CNN not only achieved an impressive PSNR but also a fair SR testing time. All models were tested in identical hardware environments, where a pretrained model was evaluated on test images of individual datasets, and their mean reconstruction times were recorded. For both datasets, the FMISR technique observed relatively swift reconstruction time. Nevertheless, the proposed method is considered the best choice because it provided overwhelming SR image quality and a satisfactory SR image acquisition time of only 0.310 and 0.296 seconds for test datasets A and B, respectively.

\section{B. SR performance by varying the FEN structure}

In this section, we analyze the performance of the SS-CNN from modifying the network structure of the FEN. For this, the network depths of both convolutional series in the FEN were varied, where multiple candidate depths in the range $M \in[3,5]$ were considered. Figs. 7(a), (b), and (c) show the modified structure of the FEN, where the depth of the symmetric convolutional series, $M$, gradually increased from 3 to 5 . To observe the effect of the depth of the convolutional series, the up-sampling layers remained intact with no structural changes. The total number of filters and their corresponding sizes were also unchanged. As the size of the network was reduced, the total number of trainable parameters was significantly reduced, which in turn decreased the training and testing times. The presented FEN with varying depths was trained with similar experimental settings, as described in Section II-E, where MSE and the Adam optimizer were used as the loss function and the optimization technique, respectively.

Fig. 8 demonstrates the reconstruction result for SR images when the network depth changed. We can see that when $M$ is 3 and 6 , the image quality deteriorated. Among the various candidates, the best PSNR performance was observed from a FEN with $M=4$. In general DL theory, higher performance from the CNNs can be attributed to the depth of the network. However, this is achieved at the cost of excessive computations and increased model complexity. Unlike this, in our study, feature map concatenation between the convolutional layers of the two symmetric series can provide sufficient feature propagation during the feed forward process. In this regard,

TABLE IV

QuANTITATIVE ANALYSIS OF THE FEN With VARIABLE NETWORK

\begin{tabular}{ccccc} 
Dataset & $M$ & $\begin{array}{c}\text { DEPTHS }(M) \\
\text { Mean } \\
\text { PSNR } \\
{[\mathbf{d B}]}\end{array}$ & $\begin{array}{c}\text { Mean } \\
\text { SSIM }\end{array}$ & $\begin{array}{c}\text { SR } \\
\text { testing } \\
\text { time }\end{array}$ \\
\hline \multirow{3}{*}{$\mathrm{A}$} & 3 & 33.28 & 0.9171 & $\mathbf{0 . 2 7 5 ~ s}$ \\
& 4 & $\mathbf{3 3 . 5 4}$ & $\mathbf{0 . 9 1 9 0}$ & $0.290 \mathrm{~s}$ \\
& 5 & 33.50 & 0.9182 & $0.293 \mathrm{~s}$ \\
& 6 & 33.44 & 0.9178 & $0.310 \mathrm{~s}$ \\
$\mathrm{~B}$ & 3 & 31.72 & 0.8653 & $\mathbf{0 . 2 6 6 ~ s}$ \\
& 4 & $\mathbf{3 1 . 9 0}$ & $\mathbf{0 . 8 6 7 7}$ & $0.284 \mathrm{~s}$ \\
& 5 & 31.67 & 0.8669 & $0.291 \mathrm{~s}$ \\
& 6 & 31.62 & 0.8668 & $0.296 \mathrm{~s}$ \\
\hline
\end{tabular}

with $M=4$, the proposed model can learn the abstract representation of the input image sufficiently well to yield HR output. On the other hand, upon increasing the depth of the FEN, we can observe degradation in the SR reconstruction performance. This can be credited to the fact that, beyond the saturation point at $M=4$, the network tends to learn redundant features due to increments in the total number of parameters in the deep model, and eventually, the quality of the reconstructed image is negatively affected. Therefore, deeper FENs do not always guarantee good performance in SR ultrasound imaging.

For a quantitative analysis of the FEN with varying $M$, mean values of the PSNR, SSIM, and SR testing time were measured. We can see from Table IV that, for both datasets, the PSNR and SSIM recorded their highest levels when $M=4$. Although shallow networks with a smaller $M$ achieved relatively shorter SR testing times, there is a trade-off in image quality. Note that the optimal $M$ is greatly influenced by the training data and the size of the input and output images. According to the requirements of image reconstruction time and complexity for the objective of SR usage, the depth of the network for an effective FEN design must be determined.

\section{CONCLUSION}

This paper explored a novel DCNN model with a symmetric series structure for SR reconstruction of ultrasound images. The proposed model comprised two parts: a FEN and an upsampling layer (for feature extraction and an up-sampling operation, respectively). The subsequent layers of the FEN were concatenated with skip connections to utilize enrichment of multi-scale deep features. The low-level feature maps obtained from both subsequent layers in the DCNN series were concatenated in a feed forward manner to support robust feature extraction and ensure high reconstruction quality in the HR output. The extracted feature maps were passed to the SPC layer, which up-sampled them by multiplying with a multidimensional kernel followed by a periodic shuffling operation to generate HR output. Simulation results showed that the proposed scheme outperformed benchmark SR models in terms of image quality, PSNR, and SSIM, and achieved a fair SR reconstruction speed. In addition, we demonstrated the influence of the FEN depth on SR reconstruction performance with ultrasound images, and proved that a FEN with a depth of 4 achieved prominent PSNR and SSIM values with the given datasets. Simulation results verified both the validity and the efficiency of the proposed DCNN-based SR model, and thus, we are convinced that the high-quality ultrasound images generated by our SR model can be applied for precise diagnosis of tumors and analysis of tissue structures.

\section{ACKNOWLEDGMENT}

This research was supported by National Research Foundation of Korea (NRF) grant funded by the Korean government (NRF-2019R1A2C4069822).

\section{REFERENCES}

[1] T. Akgun, Y. Altunbasak, and R. M. Mersereau, "Super-resolution reconstruction of hyperspectral images," IEEE Transactions on Image Processing, vol. 14, no. 11, pp. 1860-1875, Nov. 2005. 
[2] H. Shen, L. Zhang, B. Huang, and P. Li, "A MAP approach for joint motion estimation, segmentation, and super resolution," IEEE Transactions on Image Processing, vol. 16, no. 2, pp. 479-490, Feb. 2007.

[3] T. Goto, T. Fukuoka, F. Nagashima, S. Hirano, and M. Sakurai, "Superresolution system for 4K-HDTV", $22^{\text {nd }}$ International Conference on Pattern Recognition, Aug. 2014, pp 4453-4458.

[4] B. K. Gunturk, A. U. Batur, Y.Altunbasak, M. H. Hayes, and R. M. Mersereau, "Eigenface-domain super-resolution for face recognition" IEEE Transactions on Image Processing, vol. 12, no. 5, pp. 597-606, May 2003.

[5] L. Fang et al., "Deep learning-based point scanning super resolution imaging," Nat. Methods, vol. 18, no. 4, pp. 406-416, Apr. 2021.

[6] R.W. Cootney, "Ultrasound imaging: principles and applications in rodent research," ILAR Journal, vol. 42, no. 3, pp. 233-247, 2001.

[7] N. Zhao, Q. Wei, A. Basarab, D. Kouamé, and J. Tourneret, "Single image super-resolution of medical ultrasound images using a fast algorithm," IEEE 13th International Symposium on Biomedical Imaging (ISBI), Prague, Czech Republic, Jun. 2016, pp. 473-476.

[8] K. Diamantis, A. H. Greenaway, T. Anderson, J. A. Jensen, P. A. Dalgarno and V. Sboros, "Super-resolution axial localization of ultrasound scatter using multi-focal imaging," IEEE Transactions on Biomedical Engineering, vol. 65, no. 8, pp. 1840-1851, Aug. 2018.

[9] F. Zhou, W. Yang, and Q. Liao, "Interpolation-based image superresolution using multisurface fitting," IEEE Transactions on Image Processing, vol. 21, no. 7, pp. 3312-3318, Jul. 2012.

[10] Y. Zhang, Q. Fan, F. Bao, Y. Liu, and C. Zhang, "Single-image superresolution based on rational fractal interpolation," IEEE Transactions on Image Processing, vol. 27, no. 8, pp. 3782-3797, Aug. 2018.

[11] Y. Zhang, J. Liu, W. Yang, and Z. Guo, "Image super-resolution based on structure-modulated sparse representation," IEEE Transactions on Image Processing, vol. 24, no. 9, pp. 2797-2810, Sep. 2015.

[12] N. Kulkarni, P. Nagesh, R. Gowda and B. Li, "Understanding compressive sensing and sparse representation-based super-resolution," IEEE Transactions on Circuits and Systems for Video Technology, vol. 22, no. 5, pp. 778-789, May 2012.

[13] X. Mao, C. Shen, and Y.B. Yang, "Image restoration using very deep convolutional encoder-decoder networks with symmetric skip connections," 2016, arXiv: 1603.09056. [Online]. Available: https://arxiv.org/abs/1603.09056

[14] M. Haris, G. Shakhnarovich, and N. Ukita, "Deep back-projection networks for super-resolution," IEEE/CVF Conference on Computer Vision and Pattern Recognition, Salt Lake City, UT, pp. 1664-1673, Jun. 2018.

[15] W. Shi et al., "Real-time single image and video super-resolution using efficient sub-pixel convolutional neural network," Proceedings of the IEEE Conference on Computer Vision and pattern Recognition (CVPR), pp. 1874-1883, Jun. 2016.

[16] N. Ahn, B. Kang, and K.A. Sohn, "Fast, accurate, and lightweight superresolution with cascading residual network," 2018, arXiv:1803.08664. [Online]. Available: https://arxiv.org/abs/1803.08664

[17] R. C. Gonzalez, "Deep convolutional neural networks [Lecture Notes]," IEEE Signal Processing Magazine, vol. 35, no. 6, pp. 79-87, Nov. 2018.

[18] J. Lemley, S. Bazrafkan, and P. Corcoran, "Deep learning for consumer devices and services: pushing the limits for machine learning, artificial intelligence, and computer vision," IEEE Consumer Electronics Magazine, vol. 6, no. 2, pp. 48-56, Apr. 2017.

[19] K. He, X. Zhang, S. Ren, and J. Sun, "Deep residual learning for image recognition," IEEE Conference on Computer Vision and Pattern Recognition (CVPR), Las Vegas, NV, pp. 770-778, Jun. 2016.

[20] G. Huang, Z. Liu, L. Van Der Maaten, and K. Q. Weinberger, "Densely connected convolutional networks," IEEE Conference on Computer Vision and Pattern Recognition (CVPR), Honolulu, HI, pp. 2261-2269, Jul. 2017.

[21] W. Han et al., "Image super-resolution via dual-state recurrent networks," IEEE/CVF Conference on Computer Vision and Pattern Recognition, Salt Lake City, UT, pp. 1654-1663, Jun. 2018.

[22] J. S. Isaac, and R. Kulkarni, "Super resolution techniques for medical image processing," International Conference on Technologies for Sustainable Development (ICTSD), Mumbai, 2015, pp. 1-6, Feb. 2015.
[23] D. Eun et al., "Deep-learning-based image quality enhancement of compressed sensing magnetic resonance imaging of vessel wall: comparison of self-supervised and unsupervised approaches," Sci Rep, vol. 10, no. 1, pp. 1-17, Aug. 2020.

[24] Y. Zhang, and M.Q. An, "Deep learning and transfer learning based super resolution reconstruction from single medical image," Journal of Healthcare Engineering, Jul. 2017, doi:10.1155/2017/5859727.

[25] H. Liu, J. Liu, T. Tao, S. Hou, and J. Han, "Perception consistency ultrasound image super-resolution via self-supervised CycleGAN," 2020, arXiv:2012.14142. [Online]. Available: https://arxiv.org/abs/2012.14142

[26] M. Abdel-Nasser, and O.A. Omer, "Ultrasound image enhancement using a deep learning architecture," International Conference on Advanced Intelligent Systems and Informatics, pp. 639-649, Oct. 2017.

[27] S. Zhang, G. Liang, S. Pan, and L. Zheng, "A fast medical image super resolution method based on deep learning network," IEEE Access, vol. 7, pp. 12319-12327, Sep. 2019.

[28] T. Tong, G. Li, X. Liu, and Q. Gao, "Image super-resolution using dense skip connections," IEEE International Conference on Computer Vision (ICCV), Venice, pp. 4809-4817, Oct. 2017.

[29] X.J. Mao, C. Shen, and Y.B. Yang, "Image restoration using convolutional autoencoders with symmetric skip connections," 2016, arXiv:1606.08921. [Online]. Available: https://arxiv.org/abs/1606.08921

[30] Y. Sun et al., "Convolutional neural network based models for improving super-resolution imaging," IEEE Access, vol. 7, pp. 43042-43051, Apr. 2019.

[31] W. Al-Dhabyani, A. Fahmy, M. Gomaa, and H. Khaled, "Deep learning approaches for data augmentation and classification of breast masses using ultraound images," International Journal of Advanced Computer Science and Applications, vol. 10, pp. 618-627, 2019, doi: 10.14569/IJACSA.2019.0100579.

[32] M.H. Yap et al., "Automated breast ultrasound lesions detection using convolutional neural networks," IEEE journal of biomedical and health informatics, vol. 22, no. 4, pp. 1218-1226, Jul. 2018. 